Pacific Journal of Mathematics

CONJUGATE POINTS FOR NONLINEAR DIFFERENTIAL 


\title{
CONJUGATE POINTS FOR NONLINEAR DIFFERENTIAL EQUATIONS
}

\author{
Kurt Kreith and C. A. Swanson
}

Much of the classical Sturm oscillation theory has a natural generalization to linear selfadjoint differential equations of order $2 n$ if the notion of successive zeros is replaced by that of $n-n$ conjugate points. Specifically, the smallest $\beta>\alpha$ such that

$$
y(\alpha)=y^{\prime}(\alpha)=\cdots=y^{(n-1)}(\alpha)=0=y(\beta)=\cdots=y^{(n-1)}(\beta)
$$

is satisfied by a nontrivial solution of the equation is called the first conjugate point of $\alpha$ and denoted by $\eta_{1}(\alpha)$.

In the case of linear selfadjoint equations the existence of such conjugate points can be related to the existence of certain eigenvalues, and comparison theorems can be established by comparing appropriate quadratic forms. However if the equations are nonlinear or nonselfadjoint, the existence of such conjugate points cannot be established by these classical techniques. The case of fourth order nonselfadjoint linear equations was dealt with in [1] where criteria are established for assuring the existence of 2-2 conjugate points. This treatment depends on the fact that linear nonselfadjoint fourth order differential equations can be represented by second order systems of the form

$$
\begin{aligned}
& y^{\prime \prime}=a(t) y+b(t) x \\
& x^{\prime \prime}=c(t) y+d(t) x
\end{aligned}
$$

In $\S 2$ below we show how to extend this theory to nonlinear fourth order equations by considering systems of the form

$$
\begin{aligned}
& y^{\prime \prime}=f(x, y, t) \\
& x^{\prime \prime}=g(x, y, t) .
\end{aligned}
$$

For both the linear and nonlinear cases, the existence of conjugate points is related to the existence of a trajectory $x(t), y(t)$ satisfying the related second order system and

$$
y(\alpha)=y^{\prime}(\alpha)=0=y(\beta)=y^{\prime}(\beta)
$$

for some $\beta>\alpha$. The major difficulty in establishing (1.3) is in showing 
that no such trajectory can satisfy $x(t)<0$ and $y(t)>0$ for arbitrarily large values of $t$. In $\$ 3$ this particular problem is treated in a more general context of trajectories in $E^{n}$, and a number of results of independent interest are also established.

2. Fourth order equations. In this section we consider second order systems of the form

$$
\begin{aligned}
& y^{\prime \prime}=f(x, y, t) \\
& x^{\prime \prime}=g(x, y, t)
\end{aligned}
$$

where

$$
\begin{aligned}
f(x, y, t)>0 & \text { for } x>0, \\
<0 & \text { for } x<0,
\end{aligned}
$$

and

$$
g(x, y, t)>0 \quad \text { for } y>0 .
$$

For convenience $f$ and $g$ are assumed continuous on $(-\infty, \infty) \times(-\infty, \infty) \times$ $[0, \infty)$. Such systems can be used to represent a large class of nonlinear equations. Most immediately, if $f(x, y, t)=x$ and $g(x, y, t)=$ $q(t)|y|^{\mu-1} y,(2.1)$ becomes

$$
y^{(v)}-q(t)|y|^{\mu-1} y=0 \text {. }
$$

Other examples will be considered in $\S 3$.

To establish the existence of $2-2$ conjugate points relative to (2.1) we consider solutions $y(t), x(t)$ satisfying

$$
y(\alpha)=y^{\prime}(\alpha)=0 ; \quad x(\alpha)=x_{0} ; \quad x^{\prime}(\alpha)=v_{0}
$$

and seek to show the existence of at least one solution of (2.1), (2.5) satisfying

$$
y(\beta)=y^{\prime}(\beta)=0
$$

for some $\beta>\alpha$. We shall in fact establish criteria which assure the existence of such a solution for every $x_{0}>0$.

Fixing $x_{0}>0$, we may represent a solution of (2.1), (2.5) in terms of its trajectory $C\left(v_{0}\right)$ in the $x, y$-plane. The open quadrants of the plane will be denoted by I, II, III, and IV. As shown in [1] (for the linear 
case), the existence of a conjugate point trajectory satisfying (2.1), (2.5) and (2.6) follows from four conditions on the force field $\vec{F}=(g, f)$ :

(A) If for some $t_{0} \geqq \alpha$ the quantities $y\left(t_{0}\right), y^{\prime}\left(t_{0}\right), x\left(t_{0}\right)$ and $x^{\prime}\left(t_{0}\right)$ are all nonnegative (but not all zero), then $y(t), y^{\prime}(t), x(t)$ and $x^{\prime}(t)$ are all positive for $t>t_{0}$.

(B) No trajectory $C\left(v_{0}\right)$ can remain in II for arbitrarily large values of $t$.

(C) No trajectory in I satisfies

(i) $x(t) \downarrow x_{0} \geqq 0$ and $y(t) \uparrow \infty$ as $t \rightarrow \infty$,

or

$$
\text { (ii) } y(t) \downarrow y_{0} \geqq 0 \text { and } x(t) \uparrow \infty \text { as } t \rightarrow \infty \text {, }
$$

nor can any trajectory in I tend to a finite limit point $\left(x_{0}, y_{0}\right)$ in the closure of $\mathrm{I}$ as $t \rightarrow \infty$.

(D) No trajectory can go directly from II to I to II.

In order to extend these criteria to the nonlinear case under consideration, we require the following result.

LEMMA 2.1. For every $x_{0}>0$, there exists $V\left(x_{0}\right)<0$ such that $v_{0} \leqq$ $V\left(x_{0}\right)$ assures that the solution $y(t), x(t)$ of (2.1), (2.5) satisfies $y(t)<0$ for some $t>\alpha$.

Proof. From (2.2) it follows that a solution $x(t), y(t)$ of (2.1) satisfying $x(\gamma)=y(\gamma)=0 ; x^{\prime}(\gamma)<-1, y^{\prime}(\gamma)=0$ for some $\gamma>\alpha$ also satisfies $y(t)<0$ for some $t>\gamma$. By continuity and compactness we can choose constants $\gamma_{0}>\alpha$ and sufficiently small $y_{0}>0, w_{0}>0$ so that neighboring solutions $x(t), y(t)$ satisfying the conditions

$$
\begin{array}{ll}
y(\gamma)<y_{0} ; & y^{\prime}(\gamma)<w_{0} \\
x(\gamma)=0 ; & x^{\prime}(\gamma)<-1
\end{array}
$$

for any $\gamma \in\left[\alpha, \gamma_{0}\right]$ also satisfy $y(t)<0$ for some $t \geqq \gamma$. To show the existence of such neighboring solutions we note that $f(x, y, t)$ and $g(x, y, t)$ are assumed nonnegative and continuous for $(x, y, t) \in K$, where

$$
K=\left[0, x_{0}\right] \times\left[0, y_{0}\right] \times\left[\alpha, \gamma_{0}\right]
$$

Therefore there exist positive constants $f_{0}, g_{0}$ such that

$$
0 \leqq f(x, y, t) \leqq f_{0}, \quad 0 \leqq g(x, y, t) \leqq g_{0}
$$


for $(x, y, t) \in K$. Comparing the solution $y(t), x(t)$ of (2.1), (2.5) with the solution $Y(t), X(t)$ of

$$
\begin{aligned}
Y^{\prime \prime}=f_{0} ; & X^{\prime \prime}=g_{0} \\
Y(\alpha)=Y^{\prime}(\alpha)=0 ; & X(\alpha)=x_{0}, \quad X^{\prime}(\alpha)=V_{0}
\end{aligned}
$$

we conclude that for $v_{0} \leqq V_{0}$ and $(X, Y, t) \in K$,

$$
\begin{aligned}
& y^{\prime}(t) \leqq f_{0}(t-\alpha) \\
& x^{\prime}(t) \leqq V_{0}+g_{0}(t-\alpha) \\
& y(t) \leqq \frac{1}{2} f_{0}(t-\alpha)^{2} \\
& x(t) \leqq x_{0}+V_{0} t+\frac{1}{2} g_{0}(t-\alpha)^{2} .
\end{aligned}
$$

Thus for any $x_{0}>0$ it is possible to choose $V_{0}$ sufficiently negative so that for $v_{0} \leqq V_{0}$ we have $x(\gamma)=0$ for some $\gamma \leqq \gamma_{0}$ and

$$
x^{\prime}(\gamma)<-1 ; \quad y(\gamma)<y_{0} ; \quad y^{\prime}(\gamma)<w_{0} .
$$

For such choices of $v_{0}, y(t)$ will become negative as was to be shown.

There is a simple physical interpretation of this proof. Because of the uniform boundedness of $f(x, y, t)$ and $g(x, y, t)$ in $K$, one can fire a particle from $\left(x_{0}, 0\right)$ at $t=\alpha$ with a sufficiently negative initial velocity $x^{\prime}(\alpha)$ so that the particle will cross the $y$-axis at $t=\gamma<\gamma_{0}$ and satisfy

$$
x^{\prime}(\gamma)<-1 ; \quad y(\gamma)<y_{0} ; \quad y^{\prime}(\gamma)<w_{0}
$$

while crossing this axis.

On the basis of Lemma 2.1 we may assert that for every $x_{0}$

$$
\mathscr{V}_{1} \equiv\left\{v_{0} \mid C\left(v_{0}\right) \text { enters the lower half plane }\right\}
$$

is nonempty. From (2.2) and (2.3) it follows that the initial conditions $x_{0}>0, v_{0}>0$ imply that $y(t), x(t)$ will be positive for all $t>\alpha$. Therefore

$$
\mathscr{V}_{2} \equiv\left\{v_{0} \mid C\left(v_{0}\right) \text { remains in the upper half plane for all } t>\alpha\right\}
$$

is also not empty. With these properties established for (2.1), the original proof of the following result [1; Theorem 2.2] generalizes verbatim to the present nonlinear setting.

THEOREM 2.2. If conditions (A)-(D) are satisfied, then there exists a 
nontrivial solution $y(t), x(t)$ of (2.1) satisfying $y(\alpha)=y^{\prime}(\alpha)=0=y(\beta)=$ $y^{\prime}(\beta)$ for some $\beta>\alpha$.

It therefore remains to establish criteria which assure that conditions (A)-(D) are satisfied. As observed above, (A) follows easily from the integral representation of (2.1), (2.5) on account of (2.2) and (2.3). Condition (B) will be considered in $\$ 3$ in a more general setting. For the case $f(x, y, t)=x$ and $g(x, y, t)=q(t)|y|^{\mu-1} y(\mu>1)$ it will follow as a special case of Corollary 3.3 that (B) is satisfied whenever

$$
\int^{\infty} t^{\rho} q(t) d t=\infty
$$

for some $\rho<1$. Also, the first few lines of the proof of Theorem 3.1 immediately yield the following

LEMMA 2.3. For arbitrary $\epsilon>0$ suppose there exist positive-valued functions $\lambda_{\epsilon} \in C[0, \infty)$ and $\varphi \in C(0, \infty)$ such that

$$
g(x, y, t)-f(x, y, t) \geqq \lambda_{\epsilon}(t) \varphi(|x|+y)
$$

for all $(x, y) \in \mathrm{II}$ with $\min (|x|, y) \geqq \epsilon$ and for all $t \in\left[t_{0}, \infty\right), t_{0}>0$. Then condition (B) is satisfied if no solution of the differential inequality

$$
u^{\prime \prime}+\lambda_{\epsilon}(t) \varphi(u) \leqq 0 \quad \text { for any } \epsilon>0
$$

can remain positive for all $t>t_{0}$.

Lemma 2.3 is not readily applicable since explicit oscillation criteria for nonlinear differential inequalities are not well known. However, examples of explicit criteria are established in $\$ 3$, specifically in terms of (3.4) and (3.5).

Condition (C) is dealt with by means of the following.

LEMMA 2.4. If $f(x, y, t) \geqq F(x, y, t) \geqq 0$ where $F(x, y, t)$ is continuous, monotonic increasing in $x$ and $y$, and satisfies

$$
\int^{\infty} F\left(k \tau, y_{0}, \tau\right) d \tau=\infty
$$

for all constants $k>0$ and $y_{0} \geqq 0$, then no trajectory in $I$ satisfies $y(t) \downarrow y_{0} \geqq 0$ and $x(t) \uparrow \infty$ as $t \rightarrow \infty$.

Proof. If $x(t) \uparrow \infty$ then, since $x^{\prime \prime}(t)>0$ for $y>0$ by (2.1) and (2.3), 
there exists a constant $k>0$ such that $x(t) \geqq k t$ for all $t>\gamma$. Since

$$
\begin{aligned}
y^{\prime}(t) & =y^{\prime}(\gamma)+\int_{\gamma}^{t} f(x(\tau), y(\tau), \tau) d \tau \\
& \geqq y^{\prime}(\gamma)+\int_{\gamma}^{t} F(x(\tau), y(\tau), \tau) d \tau
\end{aligned}
$$

it follows that

$$
\lim _{t \rightarrow \infty} y^{\prime}(t) \geqq y^{\prime}(\gamma)+\int_{\gamma}^{\infty} F\left(x(\tau), y_{0}, \tau\right) d \tau=\infty,
$$

precluding the possibility that $y(t) \downarrow y_{0}$.

In an analogous way, trajectories satisfying $x(t) \downarrow x_{0} \geqq 0$ and $y(t) \uparrow \infty$ are precluded by the condition that $g(x, y, t) \geqq G(x, y, t) \geqq 0$ where $G(x, y, t)$ is monotonic increasing in $x$ and $y$ and satisfies

$$
\int^{\infty} G\left(x_{0}, k \tau, \tau\right) d \tau=\infty
$$

for all $k>0$ and $x_{0} \geqq 0$. The fact that $f(x, y, t)$ and $g(x, y, t)$ are positive for $x>0$ and $y>0$ precludes finite limit points in the first quadrant, and this observation, together with (2.7) and (2.8), yields criteria for (C). Finally, (D) follows as in [1] from the assumption that $g(x, y, t)>0$ for $y>0$.

By way of application of these results we shall formulate criteria which assure the existence of 2-2 conjugate points for solutions of (2.4). Other applications will follow from the results to be established in $\S 3$.

Theorem 2.5. Suppose $q(t)>0, \mu>1$, and that

$$
\int^{\infty} t^{\rho} q(t) d t=\infty
$$

for some $\rho<1$. Then the equation (2.4) has a 2-2 conjugate point $\eta_{1}(\alpha)$ for any $\alpha>0$.

Proof. Since $f(x, y, t)=x, g(x, y, t)=q(t)|y|^{\mu-1} y$ and $q(t)>0,(2.2)$ and (2.3) are clearly satisfied and (A) and (D) follow easily. From Corollary 3.4 it follows that (B) is satisfied whenever (2.9) holds for some $\rho<1$. Choosing $F(x, y, t)=f(x, y, t)=x$ and $G(x, y, t)=g(x, y, t)=$ $q(t)|y|^{\mu-1} y,(2.7)$ is automatically satisfied whereas (2.8) requires that

$$
\int^{\infty} t^{\mu} q(t) d t=\infty
$$


However since $\mu>1$ this last condition follows from (2.9). With conditions (A)-(D) so established for any $\alpha>0$, the existence of $\eta_{1}(\alpha)$ follows from Theorem 2.2.

3. Systems of nonlinear second order equations. In this section, sufficient conditions are given for the nonexistence of trajectories in an unbounded domain contained in a half-space of $n$-dimensional Euclidean space $E^{n}$. In the special case $n=2$, these conditions assure that condition (B) is fulfilled. The results obtained also constitute an essential step toward a general theory of conjugate points for even order nonlinear differential equations, and at the same time have independent interest in oscillation theory and topological dynamics.

Points in $E^{n}$ will be denoted by $x=\left(x_{1}, \cdots, x_{n}\right)$ and $\langle x, y\rangle$ will denote the inner product of $x$ and $y, x \in E^{n}, y \in E^{n}$. For a fixed $h \in E^{n}$, let $\mathscr{H}$ be the half-space

$$
\mathscr{H}=\left\{x \in E^{n}:\langle x, h\rangle>0\right\}
$$

A trajectory is defined to be the path traced out in an unbounded domain $\mathscr{S} \subset E^{n}$ by a solution vector $x$ of a vector differential equation of the type

$$
\left(a(t) x^{\prime}\right)^{\prime}=B(x, t), \quad x \in \mathscr{S}
$$

as $t$ varies over $[0, \infty)$, i.e., a trajectory is a set $\{x(t): 0 \leqq t<\infty, x$ satisfies (3.1)\}. For convenience we first deal with the case $a(t) \equiv 1$, i.e.,

$$
x^{\prime \prime}=B(x, t), \quad x \in \mathscr{S}
$$

under the assumptions listed below.

\section{Assumptions.}

1. $B: E^{n} \times[0, \infty) \rightarrow E^{n}$ is a continuous vector-valued function such that

$$
\langle-B(x, t), h\rangle \geqq \lambda(t) \varphi(\langle x, h\rangle)
$$

for all $x \in \mathscr{S}, t \in[0, \infty)$, where $h$ is a fixed $n$-vector;

2. $\lambda$ is a positive-valued continuous function in $[0, \infty)$;

3. $\varphi$ is a positive-valued $C^{1}$ function with $\varphi^{\prime}(u)>0$ in $(0, \infty)$; and either

4. $\lambda$ and $\varphi$ satisfy the conditions 


$$
\int_{c}^{\infty} \frac{d u}{\varphi(u)}<\infty, \quad \int_{1}^{\infty} t \lambda(t) d t=\infty
$$

for some number $c>0$, or that

4L. (Linear case) $\varphi(u) \equiv u$ and there exists a number $\rho<1$ such

$$
\int_{1}^{\infty} t^{\rho} \lambda(t) d t=\infty
$$

THEOREM 3.1. Under the assumptions listed above, no trajectory of (3.2) can remain in $\mathscr{S} \cap \mathscr{H}$ for all time $t$.

Proof. Suppose to the contrary that $x$ is a solution of (3.2) with $x(t) \in \mathscr{S} \cap \mathscr{H}$ for all $t>0$. Define $u(t)=\langle x(t), h\rangle$. Then $u(t)>0$ for all $t>0$, and since $x(t) \in \mathscr{S}$ it follows from (3.2) and (3.3) that $u$ satisfies the differential inequality

$$
u^{\prime \prime}=\left\langle x^{\prime \prime}, h\right\rangle \leqq-\lambda(t) \varphi(u) .
$$

Since $u^{\prime \prime}<0$ for all $t>0$, a standard argument shows that there exists a number $t_{0}$ such that $u^{\prime}(t)>0$ for all $t \geqq t_{0}$. Define

$$
v(t)=\frac{t u^{\prime}(t)}{\varphi[u(t)]}
$$

in the nonlinear case, which is a $C^{1}$ function since $\varphi[u(t)]>0$ and $\varphi \in C^{1}$ by Assumption 3. Then, using (3.6) and (3.7), we find that

$$
v^{\prime}(t) \leqq-t \lambda(t)+\frac{u^{\prime}(t)}{\varphi[u(t)]} .
$$

Integrating over $\left(t_{0}, t\right)$ we obtain

$$
v(t)-v\left(t_{0}\right) \leqq-\int_{t_{0}}^{t} \tau \lambda(\tau) d \tau+\int_{u\left(t_{0}\right)}^{u(t)} \frac{d u}{\varphi(u)},
$$

which diverges to $-\infty$ as $t \rightarrow \infty$ by the hypotheses (3.4). Therefore $v(t)<0$ for all sufficiently large $t$, and consequently $u^{\prime}(t)<0$ by (3.7). This contradicts the fact that $u^{\prime}(t)>0$ for all $t \geqq t_{0}$ and completes the proof in the nonlinear case.

In the case of linear majorization (3.3), define 


$$
v(t)=\frac{t^{\rho} u^{\prime}(t)}{u(t)}
$$

with $\rho$ as in hypothesis (3.5). Then

$$
\begin{aligned}
v^{\prime}(t) & =\frac{u(t)\left[t^{\rho} u^{\prime \prime}(t)+\rho t^{\rho-1} u^{\prime}(t)\right]-t^{\rho}\left[u^{\prime}(t)\right]^{2}}{u^{2}(t)} \\
& \leqq-t^{\rho} \lambda(t)+\rho t^{\rho-1} \frac{u^{\prime}(t)}{u(t)}
\end{aligned}
$$

However,

$$
\left(\frac{u}{u^{\prime}}\right)^{\prime}=\frac{\left(u^{\prime}\right)^{2}-u u^{\prime \prime}}{\left(u^{\prime}\right)^{2}}=\frac{\left(u^{\prime}\right)^{2}+\lambda(t) u^{2}}{\left(u^{\prime}\right)^{2}} \geqq 1
$$

so that

$$
\frac{u(t)}{u^{\prime}(t)}-\frac{u\left(t_{0}\right)}{u^{\prime}\left(t_{0}\right)} \geqq t-t_{0}
$$

Hence there exists a number $t_{1} \geqq t_{0}$ such that $u^{\prime}(t) / u(t) \leqq 2 / t$ for all $t \geqq t_{1}$, and (3.9) yields the inequality

$$
v^{\prime}(t) \leqq-t^{\rho} \lambda(t)+2 \rho t^{\rho-2}, \quad t \geqq t_{1} .
$$

Then

$$
v(t)-v\left(t_{1}\right) \leqq-\int_{t_{1}}^{t} s^{\rho} \lambda(s) d s+2 \rho \int_{t_{1}}^{t} s^{\rho-2} d s
$$

The first integral diverges by hypothesis (3.5) and the second integral converges as $t \rightarrow \infty$ since $\rho-2<-1$. Thus we again have the contradiction $v(t)<0$, so $u^{\prime}(t)<0$, for sufficiently large $t$.

COROllaRY 3.1. No trajectory of (3.2) can remain in $\mathscr{S} \cap \mathscr{H}$ for all $t>t_{0}$ if assumptions 1-3 hold for all $x \in \mathscr{S}, t \in\left[t_{0}, \infty\right)$ and if no solution $u$ of the differential inequality (3.6) can remain positive for all $t>t_{0}$.

In an earlier case considered by Kreith [1], (3.2) was a linear system $x^{\prime \prime}=A(t) x$, where $A$ denotes an $n \times n$ matrix function with continuous entries in $[0, \infty)$, i.e.,

$$
x_{i}^{\prime \prime}=\sum_{j=1}^{n} a_{i j}(t) x_{j}, \quad i=1, \cdots, n
$$


Then (3.3) is satisfied, in particular, if there exists a positive-valued continuous function $\lambda$ in $[0, \infty)$ such that

$$
-\sum_{i, j=1}^{n} a_{i j}(t) h_{i} x_{j} \geqq \lambda(t) \sum_{i=1}^{n} h_{i} x_{i}
$$

for all $x \in \mathscr{S} \cap \mathscr{H}$ and for all $t \in[0, \infty)$.

COROllary 3.2. No trajectory of (3.10) can remain in an unbounded subset $\mathscr{S} \cap \mathscr{H}$ of a half-space for all $t$ if (3.11) and (3.5) are satisfied.

A special case of interest in conjugate point theory is the case that $h=(-1,1, \cdots, 1)$ and

$$
\mathscr{S}=\left\{x \in E^{n}: x_{1}<0, x_{i}>0 \text { for } i=2, \cdots, n\right\} .
$$

Define

$$
\begin{aligned}
\epsilon_{i j} & =1 & & \text { if } i=1, j=2, \cdots, n \\
& =1 & & \text { if } j=1, i=2, \cdots, n \\
& =-1 & & \text { otherwise }
\end{aligned}
$$

$$
\lambda(t)=\min _{1 \leqq j \leqq n}\left(\sum_{i=1}^{n} \epsilon_{i j} a_{i j}(t)\right)
$$

Then

$$
-\sum_{i, j=1}^{n} a_{i j}(t) h_{1} x_{j}=\sum_{i, j=1}^{n} \epsilon_{i j} a_{i j}(t)\left|x_{j}\right| \geqq \lambda(t) \sum_{j=1}^{n}\left|x_{j}\right|=\lambda(t)\langle x, h\rangle
$$

and hence (3.11) is satisfied in the subset $\mathscr{S}$ of $\mathscr{H}$ if $\lambda(t)$ is defined by (3.12). In the case $n=2, h=(-1,1)$, the definition (3.12) reduces to

$$
\lambda(t)=\min \left[-a_{11}(t)+a_{21}(t), a_{12}(t)-a_{22}(t)\right] .
$$

We conclude that no trajectory of (3.10) can remain in the second quadrant of $E^{2}$ for all $t$ if (3.5) is satisfied, where $\lambda(t)$ is given by (3.13).

In the case $n=2$, the nonlinear system (3.2) has the form (2.1), i.e.,

$$
\left\{\begin{array}{l}
x_{1}^{\prime \prime}=g\left(x_{1}, x_{2}, t\right) \\
x_{2}^{\prime \prime}=f\left(x_{1}, x_{2}, t\right)
\end{array}\right.
$$

in the notation of this section. If $h=(-1,1)$, assumption (3.3) is 
equivalent to

$$
g\left(x_{1}, x_{2}, t\right)-f\left(x_{1}, x_{2}, t\right) \geqq \lambda(t) \varphi\left(x_{2}-x_{1}\right)
$$

for all $\left(x_{1}, x_{2}\right) \in \mathscr{S}, t \in[0, \infty)$, with $\lambda$ and $\varphi$ as in assumptions (2), (3) and (4) of Theorem 3.1. Condition (3.15) is a slight modification of that given in Lemma 2.3.

COROLlaRY 3.3. No trajectory of (3.14) can remain in $\left\{x \in \mathscr{S}: x_{2}>\right.$ $\left.x_{1}\right\}$ for all $t$ if (3.15) holds for all $x \in \mathscr{S}, t \in[0, \infty)$ and assumptions $2-4$ are satisfied.

EXAMPLE 1. Consider the Emden-Fowler prototype of (3.14):

$$
\begin{aligned}
& x_{1}^{\prime \prime}=q(t) x_{2}^{\mu}, \quad \mu>1 \\
& x_{2}^{\prime \prime}=p(t) x_{1}
\end{aligned}
$$

in II, where $p$ and $q$ are positive valued continuous functions in $[0, \infty)$ and $h=(-1,1)$ as before. The equivalent fourth order scalar differential equation is

$$
\left[\frac{1}{p(t)} y^{\prime \prime}\right]^{\prime \prime}-q(t) y^{\mu}=0, \quad \mu>1
$$

Define

$$
\begin{aligned}
\lambda(t) & =\min [p(t), q(t)], \quad t \geqq 0 \\
\mathcal{N}_{\epsilon} & =\left\{x \in E^{2}:\left|x_{1}\right|<\epsilon,\left|x_{2}\right|<\epsilon\right\}, \quad \epsilon>0 \\
\delta & =\min \left(\frac{1}{2}, \epsilon^{\mu-1}\right) \\
\mathscr{S}_{\epsilon} & =\mathrm{II}-\mathcal{N}_{\epsilon}=\mathrm{II} \cap \mathcal{N}_{\epsilon}^{c} .
\end{aligned}
$$

Then $\mathcal{N}_{\epsilon}$ is a neighborhood of the origin of fixed (but arbitrarily small) measure $\epsilon^{2}$ and $\mathscr{S}_{\epsilon}$ is all of the second quadrant except those points in $\mathcal{N}_{\epsilon}$. We assert that

$$
g\left(x_{1}, x_{2}, t\right)-f\left(x_{1}, x_{2}, t\right) \geqq \delta \lambda(t)\left(\left|x_{1}\right|+x_{2}\right)
$$

for all $x \in \mathscr{S}_{\epsilon}, t \in[0, \infty)$. To prove this, first note that in the case of (3.16)

$$
g\left(x_{1}, x_{2}, t\right)-f\left(x_{1}, x_{2}, t\right)=q(t) x_{2}^{\mu}+p(t)\left|x_{1}\right| \geqq \lambda(t)\left(x_{2}^{\mu}+\left|x_{1}\right|\right)
$$

for all $x_{2}>0, x_{1}<0$. For $x \in \mathscr{S}_{\epsilon}$, either $x_{2} \geqq \epsilon$ or $0<x_{2}<\epsilon$ and $\left|x_{1}\right| \geqq$ $\epsilon$. In the first case, the right side of (3.17) is not less than 
$\lambda(t) \min \left(1, \epsilon^{\mu-1}\right)\left(x_{2}+\left|x_{1}\right|\right)$ while in the second case it is not less than $\frac{1}{2} \lambda(t)\left(x_{2}+\left|x_{1}\right|\right)$. This proves the assertion.

Corollary 3.4. No trajectory of (3.16) can remain in the second quadrant for all $t$ if

$$
\int^{\infty} t^{\rho} \min [p(t), q(t)] d t=\infty
$$

for some number $\rho<1$.

This follows since Corollary 3.3 can be applied in $\mathscr{S}_{\epsilon}$, and $\epsilon$ is arbitrary.

EXAMPLE 2. Another special case of (3.14) is the nonlinear system

$$
\left\{\begin{array}{l}
x_{1}^{\prime \prime}=q(t) x_{2}^{\mu}+r(t)\left|x_{1}\right|^{\nu}, \quad \mu>\nu>1 \\
x_{2}^{\prime \prime}=p(t) x_{1}
\end{array}\right.
$$

where $p ; q$, and $r$ are positive valued continuous functions in $[0, \infty)$ and $h=(-1,1)$. Let $\mathscr{S}_{\epsilon}$ be as in Example 1 and define $\lambda(t)$ to be $\min [r(t), q(t)], t>0$. Then

$$
\begin{aligned}
g\left(x_{1}, x_{2}, t\right)-f\left(x_{1}, x_{2}, t\right) & =p(t)\left|x_{1}\right|+r(t)\left|x_{1}\right|^{\nu}+q(t) x_{2}^{\mu} \\
& \geqq \lambda(t)\left(\left|x_{1}\right|^{\nu}+x_{2}^{\mu}\right) \\
& \geqq \delta_{1} \lambda(t)\left(\left|x_{1}\right|^{\nu}+x_{2}^{\nu}\right) \\
& \geqq \delta_{2} \lambda(t)\left(\left|x_{1}\right|+x_{2}\right)^{\nu}
\end{aligned}
$$

for $x \in \mathscr{S}_{\epsilon}$ with $x_{2} \geqq \epsilon$, where

$$
\delta_{1}=\min \left(1, \epsilon^{\mu-\nu}\right), \quad \delta_{2}=\left(\frac{1}{2}\right)^{\nu-1} \delta_{1}
$$

in view of the standard inequality

$$
w^{\nu}+z^{\nu} \geqq\left(\frac{1}{2}\right)^{\nu-1}(w+z)^{\nu}, \quad w \geqq 0, z \geqq 0 .
$$

Also, if $0<x_{2}<\epsilon$ and $\left|x_{1}\right| \geqq \epsilon$ we have

$$
\begin{aligned}
g\left(x_{1}, x_{2}, t\right)-f\left(x_{1}, x_{2}, t\right) & \geqq \frac{1}{2} \lambda(t)\left(\left|x_{1}\right|^{\nu}+x_{2}^{\nu}\right) \\
& \geqq\left(\frac{1}{2}\right)^{\nu} \lambda(t)\left(\left|x_{1}\right|+x_{2}\right)^{\nu}
\end{aligned}
$$

by (3.20) again. It follows from (3.19) and (3.21) that 


$$
g\left(x_{1}, x_{2}, t\right)-f\left(x_{1}, x_{2}, t\right) \geqq \delta_{3} \lambda(t)\left(\left|x_{1}\right|+x_{2}\right)^{\nu}
$$

for all $x \in \mathscr{S}_{\epsilon}, t \in[0, \infty)$, where

$$
\delta_{3}=\min \left[\left(\frac{1}{2}\right)^{\nu},\left(\frac{1}{2}\right)^{\nu-1} \epsilon^{\mu-\nu}\right] .
$$

In this example, assumption (3.3) of Theorem 3.1 (or Corollary 3.3) holds in the nonlinear case $\varphi(u)=\delta_{3} u^{\nu}, \nu>1, \lambda(t)=\min [r(t), q(t)], t>0$, and hypothesis (3.4) reduces to

$$
\int^{\infty} t \min [r(t), q(t)] d t=\infty
$$

Corollary 3.5. A trajectory of (3.18) cannot remain in II for arbitrarily large $t$ if (3.23) is satisfied.

We note that the system (3.18) corresponds to the fourth order equation

$$
y^{(4)}-r(t)\left|y^{\prime \prime}\right|^{\nu}-q(t) y^{\mu}=0
$$

in the case $p(t) \equiv 1$, and corresponds to a similar fourth order equation with nonlinear damping terms when $p$ is a positive $C^{2}$ function. The results are somewhat different in the case that $r(t)$ can change sign (variable direction damping), but can be derived easily by a similar analysis. The sublinear cases $\mu<1$ and/or $\nu<1$ would require a major modification of our techniques.

An extension of Theorem 3.1 to the system (3.1) will now be given, where $a$ is a positive-valued continuous function in $[0, \infty)$. In the nonlinear case, conditions (3.4) are generalized to either conditions (3.24a) or (3.24b) below, according as the integral of $1 / a$ is divergent or convergent:

$$
\begin{aligned}
& \begin{cases}\int^{\infty} \frac{d t}{a(t)}=\infty, & \int^{\infty} \frac{d u}{\varphi(u)}<\infty, \\
\int^{\infty}\left[\int_{0}^{t} \frac{d s}{a(s)}\right] \lambda(t) d t=\infty & \int^{\infty} \frac{d u}{\varphi_{0}(u)}<\infty,\end{cases} \\
& \left\{\begin{array}{l}
\int^{\infty} \frac{d t}{a(t)}<\infty, \\
\int^{\infty} \varphi_{0}\left[\int_{t}^{\infty} \frac{d s}{a(s)}\right] \lambda(t) d t=\infty
\end{array}\right.
\end{aligned}
$$

where in (3.24b) it is assumed that the function $\varphi$ in (3.3) satisfies 


$$
\varphi(u v) \geqq \varphi_{0}(u) \varphi_{0}(v)
$$

for all $u>0, v>0$, with $\varphi_{0} \in C^{1}[0, \infty), \varphi_{0}(u)>0$, and $\varphi_{0}^{\prime}(u)>0$ in $0<u<\infty$. For example, $\varphi$ can be a power function $u \rightarrow u^{\mu}, \mu>$ 1. The last of conditions (3.24b) involves the composition of the functions $\varphi_{0}$ and $\psi$, where

$$
\psi(t)=\int_{t}^{\infty} a^{-1}(s) d s, \quad t \geqq 0
$$

The conditions in the linear case (i.e., 4L, involving (3.5)) are generalized to either (3.25a) or (3.25b) below:

$$
\begin{array}{ll}
\int^{\infty} \frac{d t}{a(t)}=\infty, & \int^{\infty}\left[\int_{0}^{t} \frac{d s}{a(s)}\right]^{\rho} \lambda(t) d t=\infty \\
\int^{\infty} \frac{d t}{a(t)}<\infty, & \int^{\infty}\left[\int_{t}^{\infty} \frac{d s}{a(s)}\right]^{\sigma} \lambda(t) d t=\infty
\end{array}
$$

for some numbers $\rho<1, \sigma>1$, respectively. We note that (3.24a) and (3.25a) reduce to (3.4) and (3.5), respectively, in the case $a(t) \equiv 1$ considered in Theorem 3.1 .

THEOREM 3.6. Under assumptions 1, 2, and 3 of Theorem 3.1 and one of (3.25a), (3.25b), (3.24a), or (3.24b), a trajectory cannot remain in $\mathscr{S} \cap \mathscr{H}$ for all time.

The proof is similar to that of Theorem 3.1 and will be omitted.

\section{REFERENCES}

1. K. Kreith, A dynamical criterion for conjugate points, Pacific J. Math., 58 (1975), 123-132.

2. C. A. Swanson, Comparison and oscillation theory of linear differential equations, "Mathematics in Science and Engineering", 48, Academic Press, New York, 1968.

Received June 9, 1976.

UNIVERSITY OF CALIFORNIA

DAVIS, CA 95616

AND

UNIVERSity of British Columbia

VANCOUVER, CANADA 


\section{PACIFIC JOURNAL OF MATHEMATICS EDITORS}

ICHARD ARENS (Managing Editor)

niversity of California

os Angeles, CA 90024

\section{. A. BEAUmont}

niversity of Washington

sattle, WA 98105

. C. MOORE

niversity of California

erkeley, CA 94720
J. DUGUNDJI

Department of Mathematics

University of Southern California

Los Angeles, CA 90007

R. FINN AND J. MiLgRAM

Stanford University

Stanford, CA 94305

\section{ASSOCIATE EDITORS}
F. BECKENBACH
B. H. NEUMANN
F. WOLF
K. YosHidA

\section{SUPPORTING INSTITUTIONS}

NIVERSITY OF BRITISH COLUMBIA

UNIVERSITY OF SOUTHERN CALIFORNIA

ALIFORNIA INSTITUTE OF TECHNOLOGY STANFORD UNIVERSITY

NIVERSITY OF CALIFORNIA

ONTANA STATE UNIVERSITY

UNIVERSITY OF HAWAII

NIVERSITY OF NEVADA

UNIVERSITY OF TOKYO

EW MEXICO STATE UNIVERSITY

UNIVERSITY OF UTAH

REGON STATE UNIVERSITY

NIVERSITY OF OREGON

WASHINGTON STATE UNIVERSITY

UNIVERSITY OF WASHINGTON

SAKA UNIVERSITY

AMERICAN MATHEMATICAL SOCIETY

The Supporting Institutions listed above contribute to the cost of publication of this Journal, but they a t owners or publishers and have no responsibility for its contents or policies.

Mathematical papers intended for publication in the Pacific Journal of Mathematics should be in typ Irm or offset-reproduced (not dittoed), double spaced with large margins. Underline Greek letters in re ierman in green, and script in blue. The first paragraph or two must be capable of being used separately as 'nopsis of the entire paper. Items of the bibliography should not be cited there unless absolutely necessary, hich case they must be identified by author and Journal, rather than by item number. Manuscripts, uplicate, may be sent to any one of the four editors. Please classify according to the scheme of Math. Revieu Idex to Vol. 39. All other communications should be addressed to the managing editor, or Elaine Bart Iniversity of California, Los Angeles, California, 90024.

100 reprints are provided free for each article, only if page charges have been substantially pai dditional copies may be obtained at cost in multiples of 50 .

The Pacific Journal of Mathematics is issued monthly as of January 1966. Regular subscription rate: \$72. year (6 Vols., 12 issues). Special rate: $\$ 36.00$ a year to individual members of supporting institutions.

Subscriptions, orders for numbers issued in the last three calendar years, and changes of address should ent to Pacific Journal of Mathematics, 103 Highland Boulevard, Berkeley, California, 94708.

UBLISHED BY PACIFIC JOURNAL OF MATHEMATICS, A NON-PROFIT CORPORATION

Printed at Jerusalem Academic Press, POB 2390, Jerusalem, Israel.

Copyright (C) 1978 Pacific Journal of Mathematics

All Rights Reserved 


\section{Pacific Journal of Mathematics}

\section{Vol. 75, No. $1 \quad$ September, 1978}

Mieczyslaw Altman, General solvability theorems

Denise Amar and Eric Amar, Sur les suites d'interpolation en plusieurs variables ..........................................

Herbert Stanley Bear, Jr. and Gerald Norman Hile, Algebras which satisfy a second order linear partial differential equation ..................

Marilyn Breen, Sets in $R^{d}$ having $(d-2)$-dimensional kernels ............

Gavin Brown and William Moran, Analytic discs in the maximal ideal space

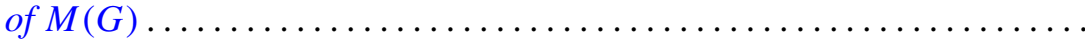

Ronald P. Brown, Quadratic forms with prescribed Stiefel-Whitney

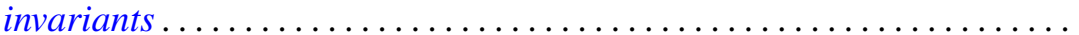

Gulbank D. Chakerian and H. Groemer, On coverings of Euclidean space by convex sets

S. Feigelstock and Z. Schlussel, Principal ideal and Noetherian groups.....

Ralph S. Freese and James Bryant Nation, Projective lattices ............

Harry Gingold, Uniqueness of linear boundary value problems for

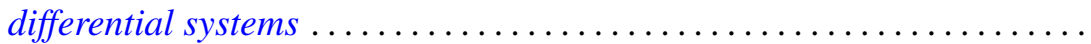

John R. Hedstrom and Evan Green Houston, Jr., Pseudo-valuation domains. . .

William Josephson, Coallocation between lattices with applications to measure extensions

M. Koskela, A characterization of non-negative matrix operators on $l^{p}$ to $l^{q}$ with $\infty>p \geq q>1$

Kurt Kreith and Charles Andrew Swanson, Conjugate points for nonlinear differential equations...........................

Shoji Kyuno, On prime gamma rings ........................ 185

Alois Andreas Lechicki, On bounded and subcontinuous multifunctions ..

Roberto Longo, A simple proof of the existence of modular automorphisms in approximately finite-dimensional von Neumann algebras ...

Kenneth Millett, Obstructions to pseudoisotopy implying isotopy for

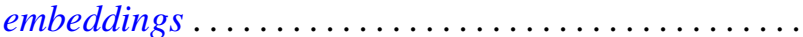

William F. Moss and John Piepenbrink, Positive solutions of elliptic equations. .

Mitsuru Nakai and Leo Sario, Duffin's function and Hadamard's

conjecture

Mohan S. Putcha, Word equations in some geometric semigroups ...

Walter Rudin, Peak-interpolation sets of class $C^{1} \ldots \ldots \ldots$

Elias Saab, On the Radon-Nikodým property in a class of locally convex

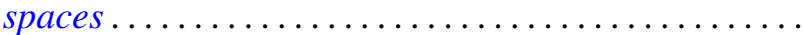

